\title{
High School Girls and Smartphone Applications to Track Menstrual Cycle
}

Tatjana Gazibara, Jelena Cakic, Milica Cakic, Anita Grgurevic, Tatjana Pekmezovic

Institute of Epidemiology, Faculty of Medicine, University of Belgrade, Belgrade, Serbia

Correspondence: pekmezovic@sezampro.rs; Tel.: + 381-11-3607-062; Fax.: + 381-11-3607-062

Received: June 26, 2020; Accepted: September10, 2020

\begin{abstract}
Objective: Tracking of periods is recommended to adolescent girls because of their irregular menstrual cycle and longer cycle length compared to adult women. The purpose of this study was to examine the prevalence of use of apps to track menstrual cycle among high school girls from Belgrade, Serbia, as well as to define factors associated with their use. Methods: A study was carried out in December 2016 and January 2017. The study included 394 high school girls from Belgrade, Serbia, who used smartphones. The students were asked to fill in an anonymous questionnaire about socio-demographic characteristics, use of online platforms and smartphone apps as well as online health (e-health) literacy scale (eHEALS). Results: The prevalence of tracking menstrual cycle via smartphone apps was $29.7 \%$. The multivariate logistic regression model showed that studying humanities-languages school program, using Wikipedia, websites run by physicians, health forums, but not YouTube were associated with use of menstrual cycle tracking apps. Girls who used fitness apps were more likely to use apps to track menstrual cycle. Conclusion: Approximately one third of girls in Belgrade highs schools who have smartphones used menstrual cycle tracking apps. Use of different digital media might influence the decision to track menstrual cycle via smartphone apps.
\end{abstract}

Key Words: Smartphone • Apps • Menstrual Cycle • Adolescents • High School.

\section{Introduction}

Almost all persons aged 16-24 years in the Republic of Serbia use mobile phones $(98.6 \%$ of males and $97.2 \%$ of females) (1). In fact, smartphones have become the preferred form of mobile phone among adolescents, because they allow access to Internet, social media, YouTube and software applications (apps) (2). Smartphone apps have been increasingly used to improve health (3) or promote healthy behaviors (4). For example, adolescents use health-related apps to manage chronic illnesses (5), achieve certain fitness (6) and diet goals (7) or track menstrual cycle (8).

Evidence suggests that girls in low and middleincome countries remain ill prepared for their first period (9). In fact, many girls continue to lack the knowledge about their menstrual cycle and repro- ductive health (9). For this reason, use of apps to track menstrual cycle allows girls and young women to better understand their body and accept their menstrual cycle as a sign of healthy functioning (10). Accounts of females who use menstrual cycle tracking apps suggest that the data provided by the apps help to prepare for the upcoming menstruation, learn about the body and period, follow-up their health status and manage fertility (11).

Tracking of periods is recommended to adolescent girls, because of their irregular menstrual cycle and longer cycle length compared to adult women (12). For this reason, menstrual cycle tracking apps are a convenient way to chart periods. While a plethora of menstrual cycle tracking apps is currently available to smartphone owners (13), studies about factors contributing to their use among adolescent girls are limited. As adolescents and young 
adults make up the vast majority of Internet users (1), we hypothesized that online health information seeking and better online health literacy of adolescents might influence their decision to use menstrual cycle tracking apps.

The purpose of this study was to examine the prevalence of use of apps to track menstrual cycle among high school girls from Belgrade, Serbia, as well as to define factors associated with their use.

\section{Methods}

The present analysis is an integral part of the study examining digital health information needs and online health information seeking in the population of high school students in Belgrade, Serbia (14).

This study was approved by the Institutional Review Board of the Faculty of Medicine, University of Belgrade. The age of majority in the Republic of Serbia is 18 years. For this reason, the schools informed the parents about study participation. The parents were offered a possibility to opt-out from the study in case they did not want their adolescent child to be included. Agreement to fill in the questionnaire was considered as assent (for participants who were minors) or consent (for adolescents aged $\geq 18$ years).

\section{Setting}

The study sample was selected from high schools in Belgrade. Duration of high school education is 4 years. High schools are oriented towards general education, but it is possible to choose between two programs: science-mathematics and humanitieslanguages, based on candidates' interests. After high school graduation, students most commonly opt to continue their education in universities. The majority of secondary schools in Serbia are public.

\section{Participants}

Because of the study purpose, only girls who owned smartphones were included in this analysis. Selection of participants is shown in Fig. 1.

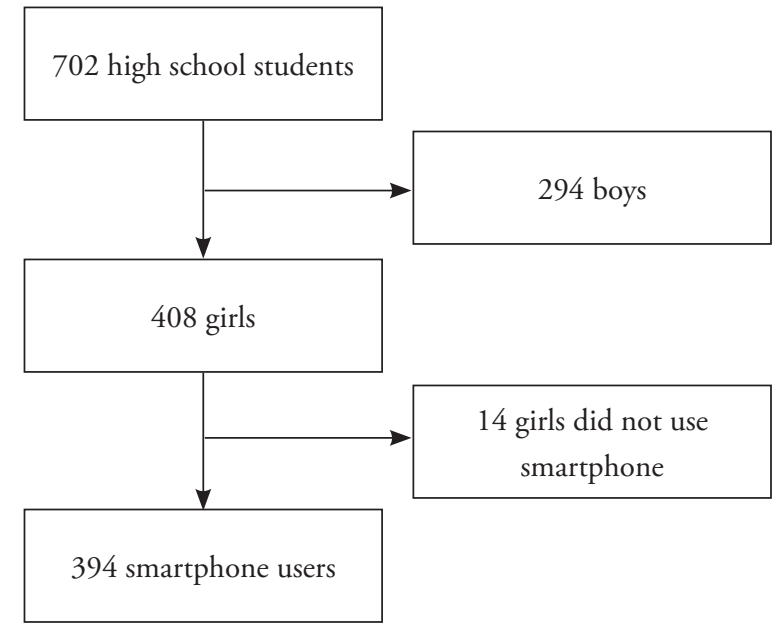

Fig. 1. Flowchart Showing the Selection of Study Participants.

Study participants were recruited from 4 out of 21 publicly funded high schools in Belgrade (16) during December 2016 and January 2017. The choice of high schools was random. Three high schools were located in the inner metropolitan area and one high school was located in the outskirts. The 4 high schools did not have the same number of classrooms (14 vs. 16). We randomly chose 7 classrooms in the two high schools with less classrooms and 8 classrooms in the two schools with more classrooms. The classrooms were selected by drawing papers slips with classrooms identifiers. The person who chose the slips was previously blinded and did not know classroom identifiers. Students from all 4 study years were represented in the study sample.

\section{Instrument}

All data were collected through a self-reported anonymous questionnaire. The questionnaire covered information about socio-demographic characteristics, such as age, type of school program [science-mathematics vs. humanities-languages], grade point average (GPA), education level of the parents and monthly income per household. The calculation of GPA was performed using a scale from 1 to 5 . The grade 1 equals "fail" and, therefore, cannot be included in the GPA at the end of 
the school year. The GPA ranged from 2.0 (pass) to 5.0 (excellent).

Education level of the parents was initially classified as primary, secondary and university level. However, because of small number of students whose parents were of primary education level, the categories 'primary' and 'secondary' education level were observed together. Monthly income per household was based on these cut-offs $<405$, 405-810 and >810 Euros per month (equivalent of Serbian dinars) according to an average monthly income in the metropolitan area of Belgrade.

We asked the students about their use of Internet and websites with health-related content. Students were asked about their use of specific websites (such as Wikipedia, health blogs, health forums, health portals, websites run by physicians, websites run by health institutions), social media and YouTube. Possible answers were dichotomous (yes vs. no).

To examine the use of smartphone apps, we first asked the students whether they used smartphones. If students answered affirmatively, they were asked to identify the apps that they used at the time of survey. The choice of apps was: 1) fitness, running, counting of steps/distance etc, 2) healthy diet, nutrition, meal preparation, calorie counting etc. and 3) menstrual cycle tracking. Use of all three types of apps was dichotomized (yes vs. no). We left an empty space for students to write the name of an app that they used in case they were unsure how to classify it. However, few students used this option.

An online health (e-health) literacy scale (eHEALS) was included in the questionnaire (17). The eHEALS is an 8-item scale, which quantifies the ability of participant to seek, comprehend and appraise online health information in order to make appropriate health care decisions (18). The answers were graded from 1 to 5 . Grade 1 equals to "strongly disagree" and grade 5 equals to "strongly agree". The grades for all 8 items are subsequently added, making the total eHEALS score. The total score ranged from 8 to 40 . Higher values corresponded to higher levels of e-health literacy. The Serbian version of eHEALS was previously tested (14). Psychometric properties of the Serbian eHEALS were adequate (14).

\section{Statistical Analyses}

All analyses were performed in the Statistical Package for Social Sciences (SPSS Inc, Chicago, IL, USA), version 20 . The level of probability $\mathrm{P}<0.05$ was considered statistically significant. The distribution of the continuous variables was not normal. For this reason, we applied Mann-Whitney test to examine the difference between the groups of girls who used and did not use apps for menstrual cycle tracking. Differences between categorical variables were tested using the Chi square test (for variables with 2 categories) and Chi square linear-by-linear association (for variables with 3 categories). Spearman's correlation coefficient rho $(\rho)$ was used to test the correlation between selected variables. To examine factors associated with use of menstrual cycle tracking apps, we used logistic regression models. The dependent variable in the model was 'use of menstrual cycle tracking apps' (yes vs. no). The independent variables were based on sociodemographic characteristics (age, school program, GPS, income, parental education level) and use of digital platforms (age at first exposure to the Internet, eHEALS score, use of Wikipedia, health blogs, health forums, health portals, websites run by physicians, websites run by health institutions, social media and YouTube). First, we ran a univariate model for each variable. The variables that were significantly or marginally significantly $(\mathrm{P}<0.25)$ associated with the dependent variable entered the multivariate logistic regression model. We tested multicollinearity of the variables in the multiple logistic regression model by evaluating the Variance Inflation Factor (VIF). The VIFs for all variables were below the cut-off value of 2.0, suggesting that multicolllinearity in this model was not an issue.

\section{Results}

The study sample included 394 high school girls who used smartphones. Of those, 117 (29.7\%) 


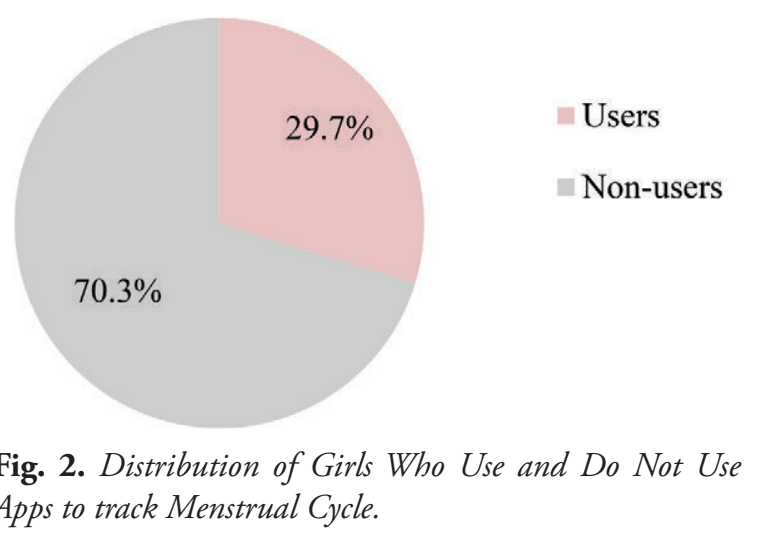

reported use of menstrual cycle tracking apps (Fig. 2). Socio-demographic characteristics and use of digital platforms according to menstrual cycle tracking apps use are presented in Table 1. Girls who used and did not use menstrual cycle tracking apps differed according to school program, household monthly income, level of parental education, use of websites run by physicians, websites run by health institutions, health forums, YouTube, fitness and diet apps (Table 1).

Use of menstrual cycle tracking apps correlated with being in humanities-languages program, high-

Table 1. Demographic Characteristics and Digital Consumer Behaviors of High School Girls According to Use of Menstrual Cycle Tracking Apps

\begin{tabular}{|c|c|c|c|}
\hline \multirow{3}{*}{ Variable } & \multicolumn{2}{|c|}{ Use of menstrual cycle tracking apps } & \multirow{3}{*}{$\mathrm{P}^{\dagger}$} \\
\hline & Yes & No & \\
\hline & $N=117 ; N(\%)$ & $N=277 ; N(\%)$ & \\
\hline Age $^{*}$ & $17.0(3.0)$ & $16.0(3.0)$ & 0.199 \\
\hline \multicolumn{4}{|l|}{ Type of school program } \\
\hline Science-mathematics & $48(41.0)$ & $156(53.3)$ & \multirow{2}{*}{0.006} \\
\hline Humanities-languages & $69(59.0)$ & $121(43.7)$ & \\
\hline Grade point average ${ }^{*}$ & $4.5(1.0)$ & $4.7(0.9)$ & 0.478 \\
\hline \multicolumn{4}{|l|}{ Household monthly income (Euros) } \\
\hline$<405$ & $10(8.5)$ & $23(8.3)$ & \multirow{3}{*}{0.025} \\
\hline $405-810$ & $42(35.9)$ & $144(52.0)$ & \\
\hline$>810$ & $65(55.6)$ & $110(39.7)$ & \\
\hline \multicolumn{4}{|l|}{ Parental education } \\
\hline Primary and secondary & $23(19.7)$ & $82(29.6)$ & \multirow{2}{*}{0.041} \\
\hline University & $94(80.3)$ & $195(70.4)$ & \\
\hline Age at first internet use ${ }^{*}$ & $10.0(4.0)$ & $10.0(3.0)$ & 0.916 \\
\hline eHEALS score ${ }^{*}$ & $27.0(7.0)$ & $26.0(9.0)$ & 0.809 \\
\hline Using Wikipedia & $48(41.0)$ & $82(29.6)$ & 0.028 \\
\hline Using websites run by physicians & $39(33.3)$ & $50(18.1)$ & 0.001 \\
\hline Using websites run by health institutions & $23(19.7)$ & 32 (11.6) & 0.034 \\
\hline Uing health blogs & $16(13.7)$ & $27(9.7)$ & 0.253 \\
\hline Using health forums & $40(34.2)$ & $54(19.5)$ & 0.002 \\
\hline Using health portals & $38(32.5)$ & $66(23.8)$ & 0.075 \\
\hline Using social media & $7(6.0)$ & $25(9.0)$ & 0.312 \\
\hline UsingYouTube & $19(16.2)$ & $76(27.4)$ & 0.018 \\
\hline Using fitness apps & $61(52.1)$ & $56(20.2)$ & 0.001 \\
\hline Using nutrition apps & $21(17.9)$ & $17(6.1)$ & 0.001 \\
\hline
\end{tabular}

*Continuous variables are presented as median (interquartile range); ${ }^{\dagger}$ For difference; ${ }^{\ddagger}$ eHealth literacy scale. 
Table 2. Correlations Between Use Of Menstrual Cycle Tracking Apps and Participants' Characteristics

\begin{tabular}{lll}
\hline Variable & $\begin{array}{l}\text { Spearman's correlation } \\
\text { coefficient }\end{array}$ & $\mathrm{P}$ \\
\hline Age & 0.065 & 0.199 \\
\hline Type of school program & 0.140 & 0.005 \\
\hline Grade point average & -0.036 & 0.479 \\
\hline Household monthly income & 0.127 & 0.012 \\
\hline Parental education level & 0.103 & 0.041 \\
\hline Age at first internet use & 0.005 & 0.916 \\
\hline eHEALS score & 0.012 & 0.809 \\
\hline Wikipedia & 0.111 & 0.028 \\
\hline Websites run by physicians & 0.167 & 0.001 \\
\hline Websites run by health institutions & 0.107 & 0.034 \\
\hline Health blogs & 0.058 & 0.254 \\
\hline Health forums & 0.158 & 0.002 \\
\hline Health portals & 0.090 & 0.075 \\
\hline Social media & -0.051 & 0.314 \\
\hline YouTube & -0.120 & 0.018 \\
\hline Fitness apps & 0.319 & 0.001 \\
\hline Nutrition apps & 0.183 & 0.001 \\
\hline
\end{tabular}

"eHealth literacy scale. er income, higher parental education level, use of Wikipedia, websites run by doctors, health forums and use of fitness and diet apps. Use of menstrual cycle tracking apps negatively correlated with use of YouTube (Table 2).

Estimates observed in the univariate logistic regression model are shown in Table 3. Studying humanities-languages program, having higher household monthly income, higher education level of parents, use of Wikipedia, websites run by physician, health institutions, health forums, fitness and nutrition apps as well as not using YouTube were univariately associated with use of menstrual cycle tracking apps. In addition to these variables, marginally significant variable (age and use of health portals) entered the final multivariate logistic regression model. The multivariate logistic regression model showed that studying humanities-languages school program, using Wikipedia, websites run by physicians, health forums, but not YouTube were associated with use menstrual cycle tracking apps (Table 3). Also, girls who used fitness apps on their smartphones was more likely to use apps to track menstrual cycle (Table 3).

\section{Table 3. Factors Associated with Use of Apps for Menstrual Cycle Tracking Among High School Girls: Results of} Univariate and Multivariate Logistic Regression Models

\begin{tabular}{|c|c|c|c|c|c|c|}
\hline \multirow{2}{*}{ Variable } & \multicolumn{3}{|c|}{ Univariate logistic regression } & \multicolumn{3}{|c|}{ Multivariate logistic regression } \\
\hline & Odds ratio & $95 \% \mathrm{CI}^{*}$ & $\mathrm{P}$ & Odds ratio & $95 \% \mathrm{CI}^{*}$ & $\mathrm{P}$ \\
\hline Age & 1.12 & $0.94-1.33$ & 0.207 & 1.11 & $0.91-1.36$ & 0.310 \\
\hline Type of school program ${ }^{\dagger}$ & 1.85 & $1.20-2.87$ & 0.006 & 1.97 & $1.20-3.25$ & 0.007 \\
\hline Grade point average & 0.89 & $0.57-1.38$ & 0.601 & - & - & - \\
\hline Household monthly income & 1.50 & $1.05-2.14$ & 0.026 & 1.04 & $0.69-1.58$ & 0.848 \\
\hline Parental education ${ }^{\ddagger}$ & 1.72 & $1.02-2.90$ & 0.043 & 1.83 & $0.99-3.37$ & 0.052 \\
\hline Age at first internet use & 0.99 & $0.90-1.09$ & 0.868 & - & - & - \\
\hline eHEALS score ${ }^{\S}$ & 1.00 & $0.97-1.04$ & 0.825 & - & - & - \\
\hline Wikipedia & 1.65 & $1.05-2.59$ & 0.028 & 1.87 & $1.11-3.16$ & 0.019 \\
\hline Websites run" & 2.27 & $1.39-3.71$ & 0.001 & 2.19 & $1.22-3.91$ & 0.008 \\
\hline Websites run & 1.87 & $1.04-3.37$ & 0.036 & 1.37 & $0.68-2.76$ & 0.372 \\
\hline Health blogs & 1.47 & $0.76-2.84$ & 0.255 & - & - & - \\
\hline Health forums & 2.14 & $1.32-3.48$ & 0.002 & 1.79 & $1.03-3.12$ & 0.039 \\
\hline Health portals & 1.54 & $0.96-2.47$ & 0.076 & 1.21 & $0.70-2.10$ & 0.492 \\
\hline Social media & 0.64 & $0.27-1.53$ & 0.316 & - & - & - \\
\hline YouTube & 0.51 & $0.29-0.90$ & 0.019 & 0.51 & $0.27-0.96$ & 0.038 \\
\hline Fitness apps & 4.30 & $2.70-6.85$ & 0.001 & 3.90 & $2.29-6.64$ & 0.001 \\
\hline Nutrition apps & 3.35 & $1.69-6.610$ & .001 & 1.97 & $0.87-4.42$ & 0.102 \\
\hline
\end{tabular}

"CI=Confidence interval; ${ }^{\dagger}$ Science-mathematics vs. Humanities-languages; ${ }^{\ddagger}$ Primary and secondary vs. University; ${ }^{\S}$ eHealth literacy scale; "By 


\section{Discussion}

To the best our knowledge, this study is the first to examine factors associated with use of menstrual cycle tracking apps among adolescent girls. The results of this study suggest that almost one third of high school girls who have smartphones use menstrual cycle tracking apps. Factors associated with use of menstrual cycle tracking apps largely account for use of various digital platforms. Higher e-health literacy was not associated with use of menstrual cycle tracking apps. Our initial hypothesis was, therefore, not supported by the study findings.

Girls who studied the humanities-languages program were more likely to use menstrual cycle tracking apps. In Serbia, most high schools have two school programs based on two different fields of study: science vs. humanities. Students opt for either one or the other school program based on their preferences and plans for further education at universities. The explanation for this finding is not entirely clear, as there are no specific differences between the two groups of high school students in the two programs except for their personal preferences about field of study.

Apps for tracking of menstrual cycle are one possible modality to track periods (19). Understanding the features of own menstrual cycle, such as the cycle length, duration of menstrual flow and menstrual volume (12), are relevant for reproductive health later in life. We assume that a decision to use menstrual cycle tracking apps is possibly a combination of recommendations from females in participants' social or peer group. It is also possible that information on online platforms influenced their decision to use menstrual cycle tracking apps, given that adolescents spend several hours daily browsing the Internet and up to $81 \%$ of teenagers report search for health-related information online (20). Worldwide, adolescent girls frequent the Internet in search of health information more commonly than boys $(21,22)$. Because adolescents are commonly being referred to as the "digital natives" (23), they frequently access various online platforms in search of health-related information.
We observed that high school girls who used Wikipedia, websites run by physician and health forums were more likely to use menstrual cycle tracking apps. It is not surprising that Wikipedia is a common health information resource on the Internet for adolescents, because other population groups, including health care workers, tend to use it as well (24). Wikipedia is accessible, usually one of the first links found using the Google search engine (24) and offers comprehensive information and references to other sources in various languages (25). Websites run by physicians, on the other hand, potentially offer a higher level of reliability of medical information, because the credentials of website editors (i.e. health professionals) ensure the accuracy of the online content. Health forums are a known digital format, where participants may remain anonymous and discuss various topics and share personal experiences. The purpose of the health forums is to engage the readers in a discussion and disseminate information (26). It is, therefore, possible that girls exchange their experiences with use of different menstrual cycle tracking apps on the forum boards. Forums are also available in local languages, which allow participation of younger adolescents who may not feel confident using English or other foreign languages.

Use of fitness apps was associated with use of menstrual cycle tracking apps in our sample of high school girls. Moreover, of all the identified factors associated with use if menstrual cycle tracking apps, the strongest effect was observed for use of fitness apps. Previous studies found that up to one third of adolescents use fitness apps $(27,28)$. Overall, fitness apps help to achieve desired fitness goals, maintain a certain level of physical activity or monitor progress (29). Combined use of fitness and menstrual cycle tracking apps is likely helpful in the efforts to organize the level of physical activity around or during menstrual cycle. Also, previous use of smartphone apps may be one of the main factors that determine the use of new apps. Finally, adolescents as "digital natives" may be more inclined to keep track and plan their activities "in one place" i.e. on their smartphone. 


\section{Strengths and Limitations}

The strength of this study is that is the first to examine factors associated with use of menstrual cycle tracking apps among adolescents girls and, therefore, provides a novel insight into practice of menstrual cycle tracking among adolescent girls. The results of this study may be used to compare the prevalence of apps use among females in other populations or older age groups. Another strength of this study is the fact that it included a representative sample of high school students from the municipalities in the inner city of Belgrade and captured a total of $1.1 \%$ of all adolescents aged $15-$ 19 years (30).

There are also certain limitations of our study. We have not asked the students whether they had their menarche nor the characteristics of their menstrual cycle. However, because the youngest girls in our sample were 15 years old, it is likely that the vast majority of girls in our sample had experienced their first period (12). We did not examine whether girls who did not use menstrual cycle tracking apps tracked their periods in other ways. Finally, this analysis was based on a cross-sectional study. The disadvantage of this methodology is limited inference about causality between the observed independent factors and the outcome.

\section{Conclusion}

In conclusion, this study showed that almost one third of girls in Belgrade highs schools who have smartphones use menstrual cycle tracking apps. Studying humanities-languages program, use of Wikipedia, websites run by physicians, health forums and fitness apps was associated with use of apps to track menstrual cycle. Use of different digital platforms might influence the decision to track menstrual cycle via smartphone apps.

Authors' Contributions: Conception and design: TG, MC, JC, AG and TP; Acquisition, analysis and interpretation of data: TG, MC, JC, AG and TP; Drafting the article: TG; Revising it critically for important intellectual content: $\mathrm{MC}$, JC, AG and TP; Approved final version of the manuscript: TG, MC, JC, AG and TP.
Acknowledgement: This survey was supported by the Ministry of Science and Technological Development of the Republic of Serbia (Grant No. 175087). The authors declare no conflict of interest.

Conflict of Interest: The authors declare that they have no conflict of interest.

\section{References}

1. Statistical Office of the Republic of Serbia. Use of information-communication technologies in the Republic of Serbia. Household, individuals, enterprises. 2017. [cited 2020 June 15] Available from https://publikacije.stat.gov. rs/G2017/Pdf/G20176006.pdf.

2. Škařupová K, Ólafsson K, Blinka L. The effect of smartphone use on trends in European adolescents' excessive Internet use. Behav Inform Technol. 2016;35(1):68-74.

3. Sage A, Roberts C, Geryk L, Sleath B, Tate D, Carpenter D. A Self-Regulation Theory-Based Asthma Management Mobile App for Adolescents: A Usability Assessment. JMIR Hum Factors. 2017;4(1):e5.

4. Dute DJ, Bemelmans WJ, Breda J. Using Mobile Apps to Promote a Healthy Lifestyle Among Adolescents and Students: A Review of the Theoretical Basis and Lessons Learned. JMIR Mhealth Uhealth. 2016;4(2):e39.

5. Mehdizadeh H, Asadi F, Mehrvar A, Nazemi E, Emami H. Smartphone Apps to Help Children and Adolescents With Cancer and Their Families: A Scoping Review. Acta Oncol. 2019;58(7):1003-14.

6. Direito A, Jiang Y, Whittaker R, Maddison R. Smartphone Apps to Improve Fitness and Increase Physical Activity Among Young People: Protocol of the Apps for IMproving FITness (AIMFIT) Randomized Controlled Trial. BMC Public Health. 2015;15:635.

7. Schoeppe S, Alley S, Rebar AL, Hayman M, Bray NA, Van Lippevelde W et al. Apps to Improve Diet, Physical Activity and Sedentary Behaviour in Children and Adolescents: A Review of Quality, Features and Behaviour Change Techniques Int J Behav Nutr Phys Act. 2017;14(1):83.

8. Fowler LR, Gillard C, Morain S. Teenage Use of Smartphone Applications for Menstrual Cycle Tracking. Pediatrics. 2020;145(5):e20192954.

9. Chandra-Mouli V, Vipul Patel S. Mapping the Knowledge and Understanding of Menarche, Menstrual Hygiene and Menstrual Health Among Adolescent Girls in Low- And Middle-Income Countries. Reprod Health. 2017;14(1):30. 
10. Adams Hillard PJ. Menstruation in Adolescents: What Do We Know? And What Do We Do With the Information? J Pediatr Adolesc Gynecol. 2014;27(6):309-19.

11. Levy J, Romo-Avilés N. "A Good Little Tool to Get to Know Yourself a Bit Better": A Qualitative Study on Users' Experiences of App-Supported Menstrual Tracking in Europe. BMC Public Health. 2019;19(1):1213.

12. Adams Hillard PJ. Menstruation in Adolescents: What's Normal? Medscape J Med. 2008;10(12):295

13. Moglia ML, Castaño PM. A Review of Smartphone Applications Designed for Tracking Women's Reproductive Health. Obstet Gynecol. 2015;125:41S.

14. Gazibara T, Cakic J, Cakic M, Pekmezovic T, Grgurevic A. eHealth and adolescents in Serbia: psychometric properties of eHeals questionnaire and contributing factors to better online health literacy. Health Promot Int. 2019;34(4):770-8.

15. United Nations Educational Scientific and Cultural Organization (UNESCO). World Data on Education. 2011. [cited 2020 June 17] Available from: http://www.ibe.unesco.org/sites/default/files/Serbia.pdf.

16. Belgrade. Secondary schools [in Serbian]. 2020. [cited 2020 June 17] Available from: http://www.beograd.rs/index.php?lang=cir\&kat=beoinfo\&sub=1528\%3F.

17. Norman CD, Skinner HA. eHEALS: The eHealth Literacy Scale. J Med Internet Res. 2006;8(4):e27.

18. Norman CD, Skinner HA. eHealth Literacy: Essential Skills for Consumer Health in a Networked World. J Med Internet Res. 2006;8(2):e9.

19. Epstein DA, Lee NB, Kang JH, Agapie E, Schroeder J, Pina LR et al. Examining Menstrual Tracking to Inform the Design of Personal Informatics Tools. Proc SIGCHI Conf Hum Factor Comput Syst. 2017;2017:6876-88.

20. Park E, Kwon M. Health-Related Internet Use by Children and Adolescents: Systematic Review. J Med Internet Res. 2018;20(4):e120.

21. Kaya N, Aşti T, Kaya I, Yaylaci S, Kaya H, Turan N et al. Association between attitudes towards Internet usage and health practices in high-school students in 2 schools in Turkey: a cross-sectional study. East Mediterr Health J. 2017;22(11):824-31.

22. Beck F, Richard JB, Nguyen-Thanh V, Montagni I, Parizot I, Renahy E. Use of the internet as a health information resource among French young adults: results from a nationally representative survey. J Med Internet Res. 2014;16(5):e128.

23. Pew Research Center. Teens, Social Media \& Technology. 2018. [cited 2020 June 18] Available from https://www. pewresearch.org/internet/2018/05/31/teens-social-mediatechnology-2018/

24. Smith DA. Situating Wikipedia as a Health Information Resource in Various Contexts: A Scoping Review. PLoS One. 2020;15(2):e0228786.

25. Heilman JM, West AG. Wikipedia and medicine: quantifying readership, editors, and the significance of natural language. J Med Internet Res. 2015;17(3):e62.

26. Hanley T, Prescott J, Gomez KU. A systematic review exploring how young people use online forums for support around mental health issues. J Ment Health. 2019;28(5):566-76.

27. De Cock N, Vangeel J, Lachat C, Beullens K, Vervoort L, Goossens L et al. Use of Fitness and Nutrition Apps: Associations With Body Mass Index, Snacking, and Drinking Habits in Adolescents. JMIR Mhealth Uhealth. 2017;5:e58.

28. Goodyear VA, Armour KM, Wood H. Young people learning about health: the role of apps and wearable devices. Learn Media Technol. 2019;44:2:193-210.

29. Direito A, Jiang Y, Whittaker R, Maddison R. Apps for IMproving FITness and Increasing Physical Activity Among Young People: The AIMFIT Pragmatic Randomized Controlled Trial. J Med Internet Res. 2015;17:e210.

30. Statistical Office of the Republic of Serbia 2011. Census of Population, Households and Dwellings in the Republic of Serbia. Population. Age and sex [in Serbian]. Data by dwellings. 2012. [cited 2020 June 19] Available from https://publikacije.stat.gov.rs/G2012/Pdf/G20124002. pdf. 